\title{
BOHR PHENOMENON FOR THE SPECIAL FAMILY OF ANALYTIC FUNCTIONS AND HARMONIC MAPPINGS
}

\begin{abstract}
In this paper we obtain the sharp Bohr radius for a family of bounded analytic functions $\mathcal{B}^{\prime}$ and for the family of sensepreserving K-quasiconformal harmonic mappings of the form $f=h+\bar{g}$, where $h \in \mathcal{B}^{\prime}$.
\end{abstract}

Key words: Bohr inequality, analytic functions, harmonic mappings, sense-preserving K-quasiconformal mappings

2010 Mathematical Subject Classification: Primary: 30A10, 30B10; 30C62, 30H05, 31A05, 41A58; Secondary: 30C75, 40A30

1. Introduction. Let $\mathbb{D}=\{z \in \mathbb{C}:|z|<1\}$ denote the open unit disk, and let $\mathcal{B}$ denote the set of all bounded analytic functions defined on $\mathbb{D}$, such that $|f(z)| \leqslant 1$ for all $z \in \mathbb{D}$. Then the classical inequality of Bohr [8] is the following:

Theorem A. Let $f \in \mathcal{B}$, with Taylor series $f(z)=\sum_{n=0}^{\infty} a_{n} z^{n}$. Then we have

$$
\sum_{n=0}^{\infty}\left|a_{n}\right| r^{n} \leqslant 1 \text { for all } r \leqslant \frac{1}{3} \text {, }
$$

where $r=|z|$.

Actually, Herald Bohr obtained in 1914 inequality (1) for $0 \leqslant r \leqslant 1 / 6$. The sharp version of the theorem in the above form was proved independently by M. Riesz, I. Schur, and F. Wiener. Other proofs were also given in [19]. Boas and Khavinson [7], and Aizenberg [3], [2] have extended the inequality to several complex variables. In [16], Kayumov et al. investigated the Bohr radius for complex-valued locally univalent harmonic mappings. Inspired by the work of [16], Evdoridis et al. [10] established several improved versions of Bohr's inequality for harmonic

(C) Petrozavodsk State University, 2020 
mappings. In [13], Kayumov and Ponnusamy discussed the Bohr radius for bounded symmetric functions and settled, in particular, the conjecture of Ali et al. [4] on the Bohr radius for the class of bounded odd functions. In [5], Alkhaleefah et al. discussed Bohr's inequality with a fixed zero coefficient and introduced the study of the Bohr phenomenon for quasisubordination family of functions. We refer the reader to the recent survey by Abu Muhanna, et al. [1], and the references therein. More information about generalizations and extensions of Bohr's result can be found in [18], [19] and in the following recent papers [4], [5], [10], [12-17]. Moreover, in [20], [21], the authors considered the problem of determining the Bohr radius in an improved form, for functions having multiple zeros at the origin. For the point of view of function spaces, we refer to [6], [9] and the references therein. Bohr's phenomenon has been investigated in different contexts, e.g., holomorphic functions of several complex variables. Since this paper deals with the classical case only, we pay attention to and cite references related to this setting.

One of important proofs of Bohr's inequality was given by Wiener, (see also [18, p. 495] and [11, p. 162]), and the following coefficient inequality plays the key role.

Theorem B. [8] Suppose that $f \in \mathcal{B}$, with Taylor series $f(z)=\sum_{n=0}^{\infty} a_{n} z^{n}$. Then

$$
\left|a_{n}\right| \leqslant 1-\left|a_{0}\right|^{2} \quad \text { for all } n \geqslant 1
$$

Note that, for all $\left|a_{0}\right| \leqslant 1$, we have $1-\left|a_{0}\right| \leqslant 1-\left|a_{0}\right|^{2}$; so, we introduce the following subset $\mathcal{B}^{\prime}$ of the set $\mathcal{B}$ :

\section{Definition 1.}

$$
\mathcal{B}^{\prime}=\left\{f: f \in \mathcal{B} \quad \text { with } \quad\left|a_{n}\right| \leqslant 1-\left|a_{0}\right| \quad \text { for all } n \in \mathbb{N}\right\}
$$

There is another good reason to consider the subfamily $\mathcal{B}^{\prime}$. In 2004, Aizenberg and Vidras (see [3, p. 736]) considered a subclass of $\mathcal{B}$ and proved the following theorem:

Theorem C. Let $f \in \mathcal{B}$, such that the Taylor coefficients $a_{m n}=0$ for $a$ given $m>1$ and all $n \geqslant 1$. Then

$$
\left|a_{n}\right| \leqslant 1-\left|a_{0}\right| \text { for all } n \geqslant 1 \text {. }
$$


In this paper, we investigate and prove Bohr's inequality for functions $f \in \mathcal{B}^{\prime}$ and, also, for sense-preserving K-quasiconformal harmonic mappings of the form $f=h+\bar{g}$, such that $\left|g^{\prime}(z)\right| \leqslant k\left|h^{\prime}(z)\right|$ in the unit disc, for $k=\frac{K-1}{K+1} \in[0,1]$ and $h \in \mathcal{B}^{\prime}$.

2. Bohr radius for a special family of analytic functions. In this section, we find the Bohr radius for a family of bounded analytic functions $\mathcal{B}^{\prime}$ and show its sharpness.

Theorem 1. Let $f(z)=\sum_{n=0}^{\infty} a_{n} z^{n} \in \mathcal{B}^{\prime}$. Then

$$
\sum_{n=0}^{\infty}\left|a_{n}\right| r^{n} \leqslant 1 \quad \text { for all } \quad r \leqslant \frac{1}{2}
$$

where $r=|z|$, and the constant $1 / 2$ cannot be improved.

Proof. Suppose that $f \in \mathcal{B}^{\prime},\left|a_{0}\right|=a$ and $|z|=r$. By the assumptions,

$$
\sum_{n=0}^{\infty}\left|a_{n}\right| r^{n}=a+\sum_{n=1}^{\infty}\left|a_{n}\right| r^{n} \leqslant a+\sum_{n=1}^{\infty}(1-a) r^{n}=a+\frac{(1-a) r}{1-r}
$$

which is, clearly, less than or equal to 1 , provided that

$$
\frac{r}{1-r} \leqslant 1, \quad \text { i. e. } \quad r \leqslant \frac{1}{2},
$$

and the first part of the theorem is completed.

To show that the constant $\frac{1}{2}$ is the best possible, let $a=\left|a_{0}\right| \in(0,1)$ and consider the function

$$
f(z)=\frac{a-\left(1-a+a^{2}\right) z}{1-a z}=a-(1-a) \sum_{n=1}^{\infty} a^{n-1} z^{n}, \quad z \in \mathbb{D} .
$$

A simple calculation shows that

$$
|f(z)| \leqslant\left|f\left(e^{i \theta}\right)\right| \leqslant 1 \quad \text { for } \quad 0 \leqslant \theta \leqslant 2 \pi
$$

whenever

$$
2 a \cos \theta-\left(2-a+a^{2}\right) \leqslant 0,
$$


which holds for all $a \in[0,1]$ and $\theta \in[0,2 \pi]$. Thus, $|f(z)| \leqslant 1$ for all $z \in \mathbb{D}$. As for the function above, note that

$$
\left|a_{n}\right|=(1-a) a^{n-1} \leqslant 1-a, \text { for all } n \geqslant 1 .
$$

So $f \in \mathcal{B}^{\prime}$. Now, for this function, we have

$$
\sum_{n=0}^{\infty}\left|a_{n}\right| r^{n}=a+(1-a) \sum_{n=1}^{\infty} a^{n-1} r^{n}=a+(1-a) \frac{r}{1-a r},
$$

which is less than or equal to 1 if $\frac{r}{1-a r} \leqslant 1$, i. e., if $r \leqslant \frac{1}{1+a}$. By making $a \rightarrow 1$, the proof of the theorem is completed.

Remark 1. By substituting $\left|a_{0}\right|^{2}$ instead of $\left|a_{0}\right|$ in the left-hand side of (5), we get

$$
\left|a_{0}\right|^{2}+\sum_{n=1}^{\infty}\left|a_{n}\right| r^{n} \leqslant 1 \quad \text { for all } \quad r \leqslant \frac{1-\left|a_{0}\right|^{2}}{2-\left|a_{0}\right|-\left|a_{0}\right|^{2}}=\frac{1+\left|a_{0}\right|}{2+\left|a_{0}\right|} .
$$

Also, observe that

$$
\inf _{0<\left|a_{0}\right|<1} \frac{1+\left|a_{0}\right|}{2+\left|a_{0}\right|}=\frac{1}{2}
$$

however, the sharpness is still an open problem. Moreover, if we substitute $\left|a_{0}\right|^{m}$ instead of $\left|a_{0}\right|$ in (5) for a fixed $m \in \mathbb{N} \backslash\{1\}$, we get

$$
\left|a_{0}\right|^{m}+\sum_{n=1}^{\infty}\left|a_{n}\right| r^{n} \leqslant 1 \quad \text { for all } \quad r \leqslant \frac{1-\left|a_{0}\right|^{m}}{2-\left|a_{0}\right|-\left|a_{0}\right|^{m}}=\frac{1+\sum_{k=1}^{m-1}\left|a_{0}\right|^{k}}{2+\sum_{k=1}^{m-1}\left|a_{0}\right|^{k}} .
$$

Again, we see that

$$
\inf _{0<\left|a_{0}\right|<1} \frac{1+\sum_{k=1}^{m-1}\left|a_{0}\right|^{k}}{2+\sum_{k=1}^{m-1}\left|a_{0}\right|^{k}}=\frac{1}{2}
$$

and, thus, the sharp Bohr radius remains open in this case, too.

Corollary 1. Suppose that $f \in \mathcal{B}$, and let $S_{N}(f, z)=\sum_{n=0}^{N} a_{n} z^{n}$ denote the partial sum of $f$, such that

$$
\left|S_{N}(f, z)\right| \leqslant 1 \quad \text { for all } \quad N \in \mathbb{N} .
$$


Then

$$
\sum_{n=0}^{\infty}\left|a_{n}\right| r^{n} \leqslant 1 \quad \text { for all } r \leqslant \frac{1}{2},
$$

Proof. The proof of this corollary follows from a result of Paulsen et al. [18]. For the reader's convenience, we include it in the form of a proposition below.

Proposition 1. [18] For any complex polynomial $p(z)=\sum_{n=0}^{N} a_{n} z^{n}$, with $|p(z)| \leqslant 1$ for all $|z|<1$ and $\left|a_{N}\right| \neq 0$, the following inequality holds:

$$
\left|a_{N}\right| \leqslant 1-\left|a_{0}\right|
$$

Example. Consider the function

$$
f(z)=\frac{a-\left(1-a+a^{3}\right) z}{1-a^{2} z}=a-(1-a) z \sum_{n=1}^{\infty} a^{2(n-1)} z^{n-1}, \quad z \in \mathbb{D},
$$

where $a \in[0,1]$. This function $f$ belongs to the class $\mathcal{B}$, because

$$
|f(z)| \leqslant\left|f\left(e^{i \theta}\right)\right| \leqslant 1 \quad \text { for all } 0 \leqslant \theta \leqslant 2 \pi
$$

if

$$
2\left(a^{2}+a-1\right) \cos \theta-(1+a)\left(2-2 a+a^{3}\right) \leqslant 0,
$$

which holds for all $a \in[0,1]$ and $\theta \in[0,2 \pi]$. The partial sum of this function is

$$
\begin{aligned}
S_{N}(f, z)=a-(1-a) z \sum_{n=1}^{N} a^{2(n-1)} z^{n-1}= & \\
& =\frac{a-\left(1-a+a^{3}\right) z+a^{2 N}(1-a) z^{N+1}}{1-a^{2} z} .
\end{aligned}
$$

Note that the inequality $\left|S_{N}\left(f, e^{i \theta}\right)\right| \leqslant 1$ holds for $N \geqslant 1$ and $\theta \in[0,2 \pi]$, since the following inequality holds:

$$
a-\left(1-a+a^{3}\right) e^{i \theta}+a^{2 N}(1-a) e^{i(N+1) \theta} \leqslant 1-a^{2} e^{i \theta} ;
$$

this means that 


$$
\begin{aligned}
2\left(a^{2}+a-\right. & 1) \cos \theta-(1+a)\left(2-2 a+a^{3}\right)+a^{4 N-1}(1-a)+ \\
& +2 a^{2 N-1}\left(a \cos ((N+1) \theta)-\left(1-a+a^{3}\right) \cos (N \theta)\right) \leqslant 0 .
\end{aligned}
$$

Let $\psi_{N}(a, \theta)$ be the expression in the left-hand side of the last inequality in (9). Note that $a^{2}+a-1 \geqslant 0$ for $a \geqslant \frac{1}{2}(\sqrt{5}-1) \backsim 0.618034$ and $1-a+a^{3} \geqslant 0$. Also note that when $\cos \theta \rightarrow 1$ and $\cos N \theta \rightarrow-1$, we get $\cos (N+1) \theta \rightarrow-1$.

Now, using the observations above for $a \geqslant \frac{1}{2}(\sqrt{5}-1)$ and $a \leqslant 1$, we conclude the following:

$$
\begin{aligned}
\psi_{N}(a, \theta) \leqslant & 2\left(a^{2}+a-1\right)-(1+a)\left(2-2 a+a^{3}\right)+(1-a)+ \\
& \quad+2\left(-a+\left(1-a+a^{3}\right)\right)= \\
= & -1-3 a+4 a^{2}+a^{3}-a^{4}
\end{aligned}
$$

which is less than or equal to zero for all $\frac{1}{2}(\sqrt{5}-1) \leqslant a \leqslant 1$, so that $\psi_{N}(a, \theta) \leqslant 0$ for all $\frac{1}{2}(\sqrt{5}-1) \leqslant a \leqslant 1$; this means that inequality (9) holds for $N \geqslant 1, \theta \in[0,2 \pi]$ and $a \in\left[\frac{1}{2}(\sqrt{5}-1), 1\right]$. Thus, $\left|S_{N}(f, z)\right| \leqslant 1$ holds for all $N \geqslant 1, z \in \mathbb{D}$, and $a \in\left[\frac{1}{2}(\sqrt{5}-1), 1\right]$.

Also,

$a+(1-a) r \sum_{n=1}^{\infty} a^{2(k-1)} r^{k-1}=a+(1-a) \frac{r}{1-a^{2} r} \leqslant 1 \quad$ for all $\quad r \leqslant \frac{1}{1+a^{2}}$, and, thus,

$$
\inf _{\frac{1}{2}(\sqrt{5}-1) \leqslant a \leqslant 1} \frac{1}{1+a^{2}}=0.5
$$

Corollary 2. Suppose that $f \in \mathcal{B}$ with the Taylor series $f(z)=\sum_{n=0}^{\infty} a_{n} z^{n}$, where $a_{m n}=0$ for all $n \geqslant 1$ and a given $m>1$. Then

$$
\sum_{n=0}^{\infty}\left|a_{n}\right| r^{n} \leqslant 1 \quad \text { for all } \quad r \leqslant \frac{1}{2} .
$$

Proof. We refer to Theorems 1 and C.

3. Bohr radius for a special family of harmonic mappings. In this section, we find the Bohr radius for the family of sense-preserving $K$ quasiconformal harmonic mappings of the form $f=h+\bar{g}$, where $h \in \mathcal{B}^{\prime}$, and show its sharpness. 
Theorem 2. Suppose that $f(z)=h(z)+\overline{g(z)}=\sum_{n=0}^{\infty} a_{n} z^{n}+\overline{\sum_{n=1}^{\infty} b_{n} z^{n}}$ is a harmonic mapping of the disk $\mathbb{D}$, such that $\left|g^{\prime}(z)\right| \leqslant k\left|h^{\prime}(z)\right|$ for some $k \in[0,1]$, and $K=\frac{1+k}{1-k}$, where $h \in \mathcal{B}^{\prime}$. Then

$$
\sum_{n=0}^{\infty}\left|a_{n}\right| r^{n}+\sum_{n=1}^{\infty}\left|b_{n}\right| r^{n} \leqslant 1 \quad \text { for all } \quad r \leqslant r_{K}:=\frac{K+1}{3 K+1},
$$

where $r=|z|$, and the constant $r_{K}$ cannot be improved.

To prove this theorem, we need the following Lemma proved in [16].

Lemma. Let $h(z)=\sum_{n=0}^{\infty} a_{n} z^{n}$ and $g(z)=\sum_{n=0}^{\infty} b_{n} z^{n}$ be two analytic functions defined in $\mathbb{D}$, such that

$$
\left|g^{\prime}(z)\right| \leqslant k\left|h^{\prime}(z)\right|
$$

in $\mathbb{D}$ and for some $k \in[0,1]$. Then

$$
\sum_{n=1}^{\infty}\left|b_{n}\right|^{2} r^{n} \leqslant k^{2} \sum_{n=1}^{\infty}\left|a_{n}\right|^{2} r^{n} \quad \text { for all } \quad|z|=r<1 .
$$

Now we return to the proof of Theorem 2 .

Proof. Consider the harmonic function $f=h+\bar{g}$, such that $\left|g^{\prime}(z)\right| \leqslant k\left|h^{\prime}(z)\right|$ in $\mathbb{D}$, where $k \in[0,1)$ and $h \in \mathcal{B}^{\prime}$. By Lemma and the Cauchy-Schwarz inequality,

$$
\begin{gathered}
\sum_{n=1}^{m}\left|b_{n}\right| r^{n} \leqslant \sqrt{\sum_{n=1}^{m}\left|b_{n}\right|^{2} r^{n} \sqrt{\sum_{n=1}^{m} r^{n}} \leqslant \sqrt{k^{2} \sum_{n=1}^{m}\left|a_{n}\right|^{2} r^{n}} \sqrt{\sum_{n=1}^{m} r^{n}} \leqslant} \\
\leqslant \sqrt{k^{2}\left(1-\left|a_{0}\right|\right)^{2} \sum_{n=1}^{m} r^{n}} \sqrt{\sum_{n=1}^{m} r^{n}}=k\left(1-\left|a_{0}\right|\right) \sum_{n=1}^{m} r^{n}
\end{gathered}
$$

Let $m \rightarrow \infty$ to get

$$
\sum_{n=1}^{\infty}\left|b_{n}\right| r^{n} \leqslant k\left(1-\left|a_{0}\right|\right) \sum_{n=1}^{\infty} r^{n}=k\left(1-\left|a_{0}\right|\right) \frac{r}{1-r},
$$


so that

$$
\left|a_{0}\right|+\sum_{n=1}^{\infty}\left|a_{n}\right| r^{n}+\sum_{n=1}^{\infty}\left|b_{n}\right| r^{n} \leqslant\left|a_{0}\right|+(1+k)\left(1-\left|a_{0}\right|\right) \frac{r}{1-r},
$$

which is less than or equal to 1 , provided that

$$
(1+k) \frac{r}{1-r} \leqslant 1, \quad \text { i. e., } \quad r \leqslant \frac{1}{2+k} .
$$

Substituting $k=(K-1) /(K+1)$, we get

$$
\sum_{n=0}^{\infty}\left|a_{n}\right| r^{n}+\sum_{n=1}^{\infty}\left|b_{n}\right| r^{n} \leqslant 1 \quad \text { for all } \quad r \leqslant \frac{K+1}{3 K+1} .
$$

This proves the first part of the theorem.

To show that the radius $\frac{K+1}{3 K+1}$ is the best possible, let $a=\left|a_{0}\right| \in(0,1)$ and consider the function

$$
\begin{aligned}
f(z) & =h(z)+\overline{g(z)}=a-\frac{(1-a) z}{1-a z}-\overline{\left(\frac{(1-a) z}{1-a z}\right)}= \\
& =a-(1-a) \sum_{n=1}^{\infty} a^{n-1} z^{n}-k(1-a) \sum_{n=1}^{\infty} a^{n-1} z^{n}, \quad z \in \mathbb{D},
\end{aligned}
$$

where $k=(K-1) /(K+1)$. Then it is very simple to see that

$$
\begin{array}{r}
\sum_{n=0}^{\infty}\left|a_{n}\right| r^{n}+\sum_{n=1}^{\infty}\left|b_{n}\right| r^{n}=a+(1-a) \sum_{n=1}^{\infty} a^{n-1} r^{n}+k(1-a) \sum_{n=1}^{\infty} a^{n-1} r^{n}= \\
=\frac{a-a^{2} r+(1+k)(1-a) r}{1-a r}
\end{array}
$$

which is bigger than or equal to 1 if and only if

$$
r \geqslant \frac{1}{1+a+k}=\frac{K+1}{2 K+a(K+1)} .
$$

Since $a$ could be chosen arbitrarily close to 1 , we obtain that the radius $\frac{K+1}{3 K+1}$ cannot be improved. The proof of the theorem is completed.

Remark 2. By Substituting $\left|a_{0}\right|^{2}$ instead of $\left|a_{0}\right|$ in the left-hand side of (12), we get

$$
\left|a_{0}\right|^{2}+\sum_{n=1}^{\infty}\left|a_{n}\right| r^{n}+\sum_{n=1}^{\infty}\left|b_{n}\right| r^{n} \leqslant 1
$$


for all $r$ with

$$
r \leqslant \frac{1-\left|a_{0}\right|^{2}}{2+k-(1+k)\left|a_{0}\right|-\left|a_{0}\right|^{2}}=\frac{1+\left|a_{0}\right|}{2+k+\left|a_{0}\right|} .
$$

We observe that

$$
r=\inf _{0<\left|a_{0}\right|<1} \frac{1+\left|a_{0}\right|}{2+k+\left|a_{0}\right|}=\frac{1}{2+k}=\frac{K+1}{3 K+1},
$$

which is an upper bound for the Bohr radius, but the sharpness case is still an open problem.

Moreover, if we substitute $\left|a_{0}\right|^{m}$ instead of $\left|a_{0}\right|$ in (12) for a fixed $m \in \mathbb{N} \backslash\{1\}$, we get

$$
\left|a_{0}\right|^{m}+\sum_{n=1}^{\infty}\left|a_{n}\right| r^{n}+\sum_{n=1}^{\infty}\left|b_{n}\right| r^{n} \leqslant 1
$$

for all

$$
r \leqslant \frac{1-\left|a_{0}\right|^{m}}{2+k-(1+k)\left|a_{0}\right|-\left|a_{0}\right|^{m}}=\frac{1+\sum_{j=1}^{m-1}\left|a_{0}\right|^{j}}{2+k+\sum_{j=1}^{m-1}\left|a_{0}\right|^{j}} .
$$

Again, we see that

$$
\inf _{0<\left|a_{0}\right|<1} \frac{1+\sum_{j=1}^{m-1}\left|a_{0}\right|^{j}}{2+k+\sum_{j=1}^{m-1}\left|a_{0}\right|^{j}}=\frac{1}{2+k}=\frac{K+1}{3 K+1}
$$

which is an upper bound for the Bohr radius in this case, too. And also, the sharpness case is still an open problem.

Acknowledgment. I would like to thank my supervisor Prof. I. R. Kayumov for useful suggestions and valuable comments and would like to thank Prof. S. Ponnusamy for fruitful discussions and suggestions.

I am very grateful to the referees for the careful reading of the paper and for their comments and detailed suggestions, which helped me to improve the manuscript considerably. 


\section{References}

[1] Abu Muhanna Y., Ali R. M., Ponnusamy S. On the Bohr inequality. In "Progress in Approximation Theory and Applicable Complex Analysis" (Edited by N. K. Govil et al.), Springer Optimization and Its Applications, 2016, vol. 117, pp. 265-295.

[2] Aizenberg L. Generalization of results about the Bohr radius for power series. Stud. Math., 2007, vol. 180, pp. 161-168.

[3] Aizenberg L., Vidras A. On the Bohr radius for two classes of holomorphic functions. Sibirsk. Mat. Zh., 2004, vol. 45, no. 4, pp. 734-746 (In Russian). http://mi . mathnet.ru/smj1103.

English version: Sib. Math. J., 2004, vol. 45, iss. 4, pp. 606-617.

DOI: https://doi.org/10.1023/B:SIMJ.0000035827.35563.b6

[4] Ali R. M., Barnard R. W., Solynin A. Yu. A note on the Bohr's phenomenon for power series. J. Math. Anal. Appl., 2017, vol. 449, no. 1, pp. 154-167.

[5] Alkhaleefah S.A., Kayumov I. R., Ponnusamy S. On the Bohr inequality with a fixed zero coefficient. Proc. Amer. Math. Soc., 2019, vol. 147, no. 12, pp. $5263-5274$.

[6] Bénéteau C., Dahlner A., Khavinson D. Remarks on the Bohr phenomenon. Comput. Methods Funct. Theory, 2004, vol. 4, no. 1, pp. 1-19.

[7] Boas H. P., Khavinson D. Bohr's power series theorem in several variables. Proc. Am. Math. Soc., 1997, vol. 125, no. 10, pp. 2975-2979.

[8] Bohr H. A theorem concerning power series. Proc. London Math. Soc., 1914, vol. s2-13, no. 1, pp. 1-5.

[9] Djakov P.B., Ramanujan M. S. A remark on Bohr's theorems and its generalizations. J. Analysis, 2000, vol. 8, pp. 65-77.

[10] Evdoridis S., Ponnusamy S., Rasila A. Improved Bohr's inequality for locally univalent harmonic mappings. Indag. Math. (N.S.), 2019, vol. 30, pp. 201-213.

[11] Garcia S. R., Mashreghi J., Ross W. T. Finite Blaschke products and their connections. Springer International Publishing, 2018.

[12] Ismagilov A., Ponnusamy S., Kayumov I. Sharp Bohr type inequality. J. Math. Anal. and Appl., 2020, vol. 486, 10 pages; Article124147.

[13] Kayumov I. R., Ponnusamy S. Bohr inequality for odd analytic functions. Comput. Methods Funct. Theory, 2017, vol. 17, pp. 679-688.

[14] Kayumov I. R., Ponnusamy S. Bohr's inequalities for the analytic functions with lacunary series and harmonic functions. J. Math. Anal. and Appl., 2018, vol. 465, no. 2, pp. 857-871. 
[15] Kayumov I. R., Ponnusamy S. On a powered Bohr inequality. Ann. Acad. Sci. Fenn. Ser. A I Math., 2019, vol. 44, pp. 301-310.

[16] Kayumov I. R., Ponnusamy S., Shakirov N. Bohr radius for locally univalent harmonic mappings. Math. Nachr., 2018, vol. 291, no. 11-12, pp. 17571768.

[17] Liu Z. H., Ponnusamy S. Bohr radius for subordination and K-quasiconformal harmonic mappings. Bull. Malays. Math. Sci. Soc., 2019, vol. 42, pp. 2151-2168.

[18] Paulsen V. I., Popescu G., Singh D. On Bohr's inequality. Proc. London Math. Soc., 2002, vol. 85, no. 2, pp. $493-512$.

[19] Paulsen V. I., Singh D. Bohr's inequality for uniform algebras. Proc. Amer. Math. Soc., 2004, vol. 132, no. 12, pp. 3577-3579.

[20] Ponnusamy S., Vijayakumar R., Wirths K.-J. New inequalities for the coefficients of unimodular bounded functions. Results in Mathematics, 2020, vol 75 , no. 107.

DOI: https://doi.org/10.1007/s00025-020-01240-1

[21] Ponnusamy S., Wirths K.-J. Bohr type inequalities for functions with a multiple zero at the origin. Comput. Methods Funct. Theory, 2020.

DOI: https://doi.org/10.1007/s40315-020-00330-z

Received February 28, 2020.

In revised form, August 04, 2020.

Accepted August 11, 2020.

Published online August 19, 2020.

Seraj A. Alkhaleefah

Kazan Federal University,

420008, 18, ul. Kremlevskaya,

Kazan, Russia

E-mail: s.alkhaleefah@gmail.com 